\title{
BREAST IMPLANT-ASSOCIATED ANAPLASTIC LARGE CELL LYMPHOMA (BIA-ALCL): A CASE REPORT WITH ATYPICAL SYMPTOMS
}

Elisana Maria Santos Caires ${ }^{1}$, Régis Resende Paulinelli' ${ }^{1}$ Miliana Tostes Lucattoํ, Eneida Ribeiro Marinho, Henrique Moura de Paula ${ }^{1}$

${ }^{1}$ Hospital Araújo Jorge - Goiânia (GO), Brazil.

The BIA-ALCL is a rare type of T-cell lymphoma CD30+ e AKL-, occurring more common in women with Allergantextured implants. It presents most frequently as a late-onset accumulation of seroma fluid between the implant and less frequently as a palpable tumor mass, with malignant cells infiltrating through the capsule and surrounding tissue with potential lymph node and systemic involvement. This article describes a case report of a 65-year-old female patient with BIA-ALCL complaining of erythema in her right breast for almost 7 months. She agreed no family history of cancer and no fever. The patient was diabetic type 2, dyslipidemic, and postmenopausal taking estrogen therapy. She had undergone a breast augmentation with $215 \mathrm{~mL}$ polyurethane-coated implants 15 years ago. Imaging revealed right axillary lymph node enlargement, thickening of the breast parenchyma, and minimal periprosthetic seroma. The initial suspicion was infection wherefore she was submitted removal of the implants, partial capsulectomy on the right side, and total contralateral capsulectomy. Immunochemistry confirmed BIA-ALCL CD30+ e AKL- on the right and no disease on the left. Bacterioscopy was negative. A complementary surgical procedure involving removal of all the right capsules, resection of axillary palpable nodes, and reconstruction was necessary to achieve a bilateral oncoplastic mastopexy. The final diagnosis was BIA-ALCL confined capsule with negative margins and none axillary lymph nodes involvement, staging IB. No adjuvant treatment was necessary. The patient remained symptom free during follow-up examinations, and she desires a new breast augmentation.

Keywords: Breast implant-associated anaplastic large cell lymphoma; Breast implants; Lymphoma. 